\title{
The Importance of Mental Representations in Design Engineering
}

\author{
Brad Crowell \\ Department of Electrical \\ \& Computer Engineering \\ Dalhousie University \\ Halifax, N.S. \\ crowell@amirix.com
}

\author{
Dr. Peter Gregson \\ Department of Electrical \\ \& Computer Engineering \\ Dalhousie University \\ Halifax, N.S. \\ Peter.Gregson@Dal.Ca
}

\begin{abstract}
At its fundamental level, engineering is problem solving. An engineer must have problem solving abilities with some level of expertise. However, gaining these skills is challenging since design is often viewed as more of an art than science. This paper investigates the cognitive aspects of design expertise.

We conclude that expertise relies on knowledge of the basic principles and the application of this theory. Examining the differences between expert and novice problem solving highlights this. Fundamental to the differences in problem solving ability and strategies is the level of sophistication, appropriateness, and completeness of their mental representations of the problem.
\end{abstract}

\section{Introduction}

Engineering practice is a broad description of many activities within various disciplines; mechanical, electrical, structural, and industrial to name a few. There are differences between each of these disciplines, but there is one aspect of the engineering profession that spans these very different fields. Engineering practice is problem solving. It involves using various skills that are based on applied science, derived from the fundamental science of the underlying discipline, to provide a service for a client [1].

Good design has been classified as an art, an ability that not everyone has or can develop. More modern approaches to this subject have proposed a scientific approach to the "art of design", but this new "science of design" has not yet been fully developed [1].
It is difficult to determine how an engineer with many years of experience approaches the details of design decisions when they have trouble expressing their own reasoning. They don't know exactly why they choose one path over another, but their results, when solving a difficult problem or providing a creative idea, speak for themselves. The internalization of knowledge by the expert prevents $\mathrm{s} / \mathrm{he}$ from conveying the process of design [2]. Thus, notions of art and mystique continue to surround expert engineering design. However, considerable progress has been made in investigating the cognitive aspects of knowledge and expertise to reveal the basic principles of expert design.

\section{Cognitive Psychology}

Cognitive psychology is the study of thinking, in its most general terms. Cognition includes "the interpretation, manipulation, and transformation of mental representations" [3]. A great variety of studies have been completed in attempts to understand how we understand, learn, and use knowledge. Of particular interest for this paper are the studies of expertise.

\section{Expertise}

Expertise represents the highest level of cognitive skill [2]. Studies of expertise have been interested in the storage, organization, and use of domain-specific information by experts, as compared to novices [2]. To an observer outside the specific domain, expertise is more of an art with the associated mystique, than the application of logic. Expert knowledge appears as something inexplicably obtained through many years of experience by someone with above average intellect. Although an ego booster for the expert, the idea of expert intuition 
has been shown to be only an impression on the part of the non-expert [2].

Expertise comes from a vast knowledge base that is specific to the domain of interest and is very well organized. In fact, the more complex a domain is, the more organized this knowledge must be [2] The "art" that an expert exhibits is due to his greater knowledge that is not apparent to the observer and in the differences between the problem representation used by the expert and his description of it [2]. The expert's mental model can be more sophisticated than even they can describe. This level of sophistication is the first significant difference between experts and novices.

It will take many years to obtain and properly organize the required amount of information, particularly if it is not a directed process, which is the usual case for practicing engineers [2]. Perhaps a more focused approach, aimed at restructuring and interconnecting the individual pieces of the basic knowledge within a domain, may help to reduce the time needed to obtain expertise.

Most studies of expertise have focused on the differences between experts and novices. These differences include their use of knowledge, which can take three forms; accretion, the amassing of new knowledge; tuning, adapting to changes in knowledge; and restructuring, the reorganization of knowledge [2].

The retrieval of knowledge by experts has been found to be less susceptible to interference while their performance is more automated and internalized. In many cases, the knowledge the expert holds is so internalized and procedural, that he is not able to explain his reasoning [2]. Experts also tend to change strategies as needed. Expert knowledge is believed to be organized categorically, but with a finer granularity than for the novice. Even with a great amount of domain-specific information stored in memory, experts have demonstrated better and more efficient retrieval [2].

\section{Expertise in Chess and Medicine}

Chess players have been studied quite often in relation to expertise [4]. The ability of expert chess players to remember the orientation of pieces on a chessboard has been shown to be quite superior, in both recall and the memorization time required, to that of novices when real game situations are used. Interestingly, random positioning of the pieces resulted in little difference between the expert and novice [4]. As chess players gain experience, they store in their memory chunks or clusters representing common game positions. Thus, when faced with a real chess game, they are better able to connect the present positions with those of previously played games, thereby aiding recall. No such chunking advantage is available when facing randomly placed pieces [4]. However, chunking theory does not appear to account for the total number of pieces experts are able to manage. Along with chunking, it is now proposed that templates and retrieval structures are used. Templates are used for large organizations of pieces within which information from chunks can be arranged [4].

Similar results have been found in studies of medical practitioners. Medical experts were found to have reduced recall of normal x-rays, compared to novices. Differences in general memory recall was ruled out and this reduced expert recall was attributed to the lack of important instances to aid retrieval of the information. The experts took less notice of the ordinary aspects of these normal x-rays [4].

\section{Expertise in Physics}

Another domain under considerable study for expertise has been physics. When asked to sort various physics problems, experts were found to associate problems based on the basic principles of the domain knowledge used in the solution, rather than on the more superficial sorting criteria of the novices [4]. When solving problems, experts tended to take longer to begin their solution than the novices [4]. Furthermore, most experts used a forward chaining method, from the basic principles of the domain to the solution, while novices began with the goal and attempted to match this to an appropriate principle. In a subsequent study, while experts and novices used forward chaining solution methods, experts devised more detailed plans than the novices before beginning the solution [4].

\section{Expertise in Computer Programming}

Expertise has also been investigated within the domain of computer programming. Syntactic, semantic, schematic, and strategic are the basic types of knowledge identified for debugging software programs. Syntactic knowledge encompasses the syntax rules of the programming language used, the factual knowledge of the domain. The mental model constructed of the major structures, objects, and functions defined in the code, as well as those of the computer itself, comprise the semantic knowledge. The programmer's schematic knowledge is made up of their catalog of routines and algorithms for achieving particular tasks. Finally, strategic knowledge, which is procedural, is used to develop plans and to monitor the proposed solution [5].

Expert programmers have internalized their syntactic knowledge and are able to debug 
programming language errors with minimal cognitive effort. As would be expected, novices have to focus more cognitive energy on finding syntax issues within code segments. This automation of syntactic knowledge allows the expert to use more cognitive resources to address the higher level programming issues [5]. Furthermore, chunking of information is a large benefit for expert programmers. This ability to reduce a code segment from many lines to five or six common chunks of statements decreases their cognitive load [5].

A problem-sorting study of novice and expert programmers to investigate their schematic knowledge revealed similar results to the physics problem-sorting study. The cover stories for the programming problems defined the sorting criteria for the novices, while experts demonstrated the use of their schematic knowledge by using the procedure type required in the solution as the basis for sorting $[5]$.

In solving programming problems, both the experts and novices subdivided the posed problem into smaller components, but a more systematic approach was demonstrated by the experts [5]. Similarly, both programmer types planned their solutions, but the experts entertained more solution options [5].

When debugging, experts demonstrated a global planning strategy through their tendency to take more time to acquaint themselves with the code segment before editing. Novices attempted to solve problems before gaining a full understanding of the program. They paused often, indicating a local planning method [5]. This also indicates that experts may be waiting until they have a complete mental representation of the problem before attempting a solution, the second important difference between experts and novices. Furthermore, experts employed a top down approach as they concentrated on high level functions in debugging [5]. Novices were less focused in their approach as they skipped around different parts of the code. In both debugging and writing tasks, experts use higher level strategies than novices $[5]$.

A lack of semantic knowledge has been identified in many novice programmers. This deficiency limits their ability to develop optimal code sequences. In a study of 618 Pascal inexperienced programmers, students were given either a traditional Pascal course or one that focused on providing a definitive model of the computer [6]. A computer program, Paslab, was developed to give students a graphical overview of the inner workings of the computer. As well, this program allowed students to experience "the way in which an expert programmer proceeds" [6, pg 329]. Thus, this view into expertise enhanced the student's mental model of the computer, making it more appropriate, complete, and sophisticated than in the traditional course.

A predictor of success in the traditional course was previous programming experience with another language, in particular BASIC [6]. However, the Paslab based course did not show a similar result. In this course, the student's mind-set toward the course, rather than previous experience, was correlated to student success. Of note was that student anticipation of the course being hard was a primary indicator [6]. Also of particular importance was that $23 \%$ of students failed the traditional course while only $11 \%$ failed the Paslab course [6]. Interestingly, little change was seen at the highest level of student achievement, $22 \%$ and $21 \%$ of students achieved the grade of distinction for the traditional and Paslab course respectively [6]. Thus, the use of Paslab tended to help those students having difficulty, enabling them to be raised from the failing group to join those with more satisfactory results [6]. Furthermore, students in the Paslab course rated the assignments as harder than those in the traditional course. However, they tended to overcome the challenges presented, while those students in the traditional course having problems with the assignments were noted as unable to learn programming [6]. It is apparent that the better mental model of the Paslab students provided them the means to solve the assignment problems and learn in the process.

\section{Expert Problem Solving}

The quality of problem solving within a domain is extremely dependent upon both the quality and quantity of domain knowledge possessed by the solver. The differences in knowledge between novice and expert programmers lie in their syntactic, semantic, schematic, and strategic knowledge [5]. Experts store syntactic knowledge in larger pieces, allowing faster access. Their links between problem elements and their semantic knowledge are stronger than in novices. Furthermore, the expert's schematic knowledge allows better classification of problems that directly leads to a potential solution. Finally, experts strategically work forward in problem solving using global planning and an openness to considering multiple options [5].

Theories of problem solving differences between experts and novices have been developed from studies that overwhelmingly use problems that are routine for the experts. More study of how experts solve unfamiliar and difficult problems is needed to fully understand expertise [7]. Some indication has 
been given that when confronted with a new problem, experts do use backward chaining methods in their solution attempts. To effectively work forward requires certain domain-specific rules be known and used. If those rules are not known, either by a novice or an expert, a backward chaining solution may be used [7]. When the conversion is made from backward to forward chaining, and what promotes this change, as expertise is gained also remains to be determined [7]. Of note are the recurring adjustments that occur to semantic knowledge as expertise is gained. As this knowledge is applied to various problems, this episodic knowledge is linked to the underlying concepts providing the expert with additional information on how to best use the semantic knowledge. Through this incremental learning, knowledge is refined and expanded. When knowledge is applied to problems and time is taken to reflect on its success or failure, this refinement of knowledge is enhanced [7]. Incremental learning and reflection provide the means to ensure the appropriateness of the mental model, which is the third significant difference between novices and experts.

Through these observations of expert and novice problem solving it is apparent that knowledge of the basic principles of the domain and the interconnections between this knowledge and the practical applications are the crucial differences in problem solving performance. The importance of fundamental domain knowledge was emphasized by Ryder who stated, "new knowledge is developed by those with the most fundamental and appropriate knowledge and in any technical field, those with the best fundamental education will lead in the advance of the field" [as cited in 8, pg 113].

\section{Mental Representations}

Mental representations have been discussed briefly, however in light of the importance of this subject a more in-depth discussion is warranted. Mental representations are fundamental to problem solving performance and underlie the main differences between experts and novices.

Mental representations have two very important features. First, they provide the means to store information and understanding of objects and events. Second, they also provide mental processes that can act within the representational system [3].

\section{Naïve, Qualitative, and Quantitative Representations}

Of particular interest is a study that proposes three types of representations developed from the physics domain; naïve, qualitative scientific, and quantitative scientific representations [9].

Naïve representations use common sense knowledge drawn from the environment. Through envisioning or qualitative simulation, the evolution of the problem is considered over time [9].

A qualitative representation uses abstract knowledge of concepts combined with knowledge of when and where these concepts can properly be applied. It also includes the features of the objects and the ranges of values that would be appropriate for the particular situation [9].

The mathematical equations, algebraic or vector, that precisely describe the concepts of the domain make up the quantitative representation. The quantification of knowledge occurs in levels or stages. At the simplest level, a relationship between concepts is known. The next higher level within this hierarchy includes a more detailed relationship. As the quantitative hierarchy is traversed, the representation becomes more precise until finally, the description is the complete algebraic equation [9].

The importance of both qualitative and quantitative domain knowledge representations can be seen in problem solving activities. Primitive problem solvers do not always use quantitative and qualitative representations appropriately [9]. In these cases, the solver uses the equations in a backward chaining search to attempt to find the solution. However, very often the solution evades them because of the difficulty in managing the various equations and the proper application of them [9]. More successful problem solvers demonstrate closely linked quantitative and qualitative knowledge. Their problem solving process involves recurring stages of qualitative and quantitative analysis applied in a forward chaining manner, obtaining additional information until the solution for the desired variables are complete [9].

This study was extended to include the development of a computer program, Sepia, to simulate the cognitive processes using qualitative and quantitative knowledge of the physics domain. Without access to qualitative knowledge, Sepia demonstrated the primitive techniques of novice problem solvers, applying the various equations available to find the appropriate one that may lead to a solution. When provided both quantitative and qualitative knowledge, a more complex and successful problem solving approach was demonstrated [9]. 
The Sepia program models problem solving competence of three basic types of problems, each differentiated by the knowledge used. The first uses only quantitative knowledge in problem solving and in general does not succeed in deriving the solution [9]. The second problem type uses both quantitative and qualitative knowledge that is both correct and complete. In this case, the program alternates between the use of quantitative and qualitative knowledge, defining constraints and preconditions through the use of qualitative knowledge that are applied to the use of quantitative knowledge. Qualitative knowledge is also useful in making explicit the information that is not stated in the problem definition but nonetheless appropriate and important to the solution. Furthermore, qualitative knowledge can be used to construct additional equations applicable to the particular problem, thereby broadening the quantitative knowledge available [9]. The final type of problem modeled by Sepia uses both quantitative and qualitative knowledge. However, the qualitative knowledge, although accurate, is incomplete. In most cases, this situation results in either an incorrect or only a partial solution to the problem [9].

This study suggests that the lack of qualitative knowledge in problem solving is the cause of many student errors. Furthermore, an increase in problem solving ability results from the teaching of qualitative knowledge [9].

These knowledge representations can be applied to the understanding of the difference between novices and experts. Novices often demonstrate inadequate and/or unlinked qualitative knowledge [9]. In contrast, experts have constructed sophisticated domain knowledge representations that tightly link their qualitative and quantitative knowledge [9]. The restructuring of the qualitative and quantitative knowledge to tightly integrate this information is essential to problem solving [9].

\section{Conclusions}

In view of the extreme importance of mental representations to problem solving performance, and therefore engineering design, developing a model with both quantitative and qualitative knowledge is vital. Without the appropriate qualitative knowledge, problem solving based on a quantitative mental representation alone is destined to fail. Bringing to bear even partial qualitative knowledge significantly increases problem solving ability. This highlights the differences between the mental representations used by experts and novice problem solvers:

- Sophistication

- Completeness

\section{- Appropriateness}

The expert develops a more sophisticated mental representation using his extensive domain knowledge, effectively linking his quantitative and qualitative knowledge. The various aspects of the problem are investigated to incorporate all relevant information into a complete mental representation before attempting to solve the posed problem. Finally, qualitative knowledge is used to refine the mental model and ensure its appropriateness to the problem at hand.

Gaining expertise is the goal of every practicing engineer and student. Unfortunately, achieving this level of expertise has been left as a side effect of professional practice and technical education, rather than a directed goal. Furthermore, the concept of prerequisite knowledge, prevalent in formal education, requires that knowledge be gained in a fixed sequence. This results in students acquiring fragmented pieces of information, without the tight connections between this knowledge, which is so important [9]. In addition, when the purpose of learning particular knowledge is not known by the student, there is less motivation than when a specific purpose is apparent [9].

The common theme that becomes evident when addressing expertise is the mastery of the basic principles and connections between this theory and professional practice within the domain. The quantity and quality of fundamental domain knowledge directly affects the sophistication, completeness, and appropriateness of the resulting mental representations. Thus, providing this foundation is vital in undergraduate education. However, the links between these basic principles and their practical application are equally important. To attain expertise, the technical education of an engineer and the subsequent years of professional practice must be focused on obtaining the qualitative and quantitative domain knowledge and forming the knowledge interconnections.

\section{References}

[1] Schön, Donald A.; The Reflective Practitioner: How Professionals Think in Action; Basic Books Inc. New York; 1983.

[2] Gavin, Helen; The Essence of Cognitive Psychology; Prentice Hall Europe; 1998.

[3] Sternberg, Robert J.; Thinking and Problem Solving: Handbook of Perception and Cognition; Academic Press Inc.; 1994. 
[4] Parker, Alan J.; Essential Cognitive Psychology; Psychology Press, 2000.

[5] Mayer, Richard E.; Thinking, Problem Solving, Cognition; WH Freeman and Company; 1992.

[6] Goodwin, Leanard, and Sanati, Mohammad; Learning Computer Programming Through Dynamic Representation of Computer Functioning: Evaluation of a New Learning Package for Pascal; International Journal of Man-Machine Studies 25, pg. 327-341, Academic Press Inc, 1986.

[7] Gilhooly, K. J.; Human and Machine Problem Solving; Plenum Press; 1989.

[8] Ghosh, Sumit; An Exercise in Inducing Creativity in Undergraduate Engineering Students Through Challenging Examinations and Open-Ended Design Problems; IEEE Transactions on Education, Vol. 36, No 1, pg. 113-119, February 1993.

[9] Ploetzner, Rolf and Spada, Hans; Multiple Mental Representations of Information in Physics Problem Solving; in: Strube, Gerhard; Wender, Karl F.; The Cognitive Psychology of Knowledge; Elsevier Science Publishers; pg. 289-309, 1993. 